\title{
EL EMPOWERMENT: UNA PODEROSA HERRAMIENTA CAPAZ DE TRANSFORMAR ORGANIZACIONES
}

\author{
José Román ${ }^{1}$ \\ Célia Ribeiro ${ }^{2}$
}

Resumo: As empresas ou organizações têm um elemento comum: todas são constituídas por pessoas, que constituem o seu capital humano, a sua energia vital, assegurando a sua capacidade de transformação.

Os meios técnicos e as infraestruturas são importantes, mas, em última análise, são as pessoas que levam a cabo os desenvolvimentos e as mudanças a favor das organizações para se adaptarem ao seu ambiente e se tornarem competitivas.

O contexto atual globalizado ao nível da economia, tecnologia, mercado e recursos humanos, altamente competitivo, mais não fez do que reforçar o valor das pessoas, convertendo-as no elemento que, estando motivadas, empoderadas, e sob uma liderança dinamizadora, é capaz de potenciar a capacidade inovadora das empresas. É aí que nasce o empowerment, como ferramenta capaz de libertar o poder das pessoas, convertendo-as em parceiras da organização.

Palavras-chave: Pessoas, mudança, competitividade, empowerment.

Abstract: Companies or organizations have a common element: they are all composed by people, who represent their human capital, their vital energy and ensure their capacity of change.

Technical devices and infrastructures are important, but, ultimately, it is people who carry out the developments and changes in favour of organizations, so that they may adapt to their environment and become competitive.

At economic, technological, market and human resources levels, the globalized context of nowadays is highly competitive and merely

\footnotetext{
${ }^{1}$ Mestre em Gestão, Especialização em Gestão de Negócios. Professor Universitário da Área de Gestão, Gestor da Área de Estratégia e Marketing. E-mail: roman.jose07@gmail.com

2 Doutorada em Psicologia, área de especialidade em Psicologia Pedagógica. Professora Auxiliar da Universidade Católica Portuguesa - Viseu. E-mail: cribeiro@viseu.ucp.pt
} 
reinforces the value of people. Being motivated, empowered and under a dynamic leadership, people become the element that is capable of enhancing the innovative capacity of companies. Empowerment rises here as a tool that allows to unleash the power of people, converting them into partners in the organization.

Keywords: People, change, competitiveness, empowerment.

\section{INTRODUCCIÓN}

Ante la complejidad de la creciente competencia sumada al incremento de la demanda y las exigencias del mercado en cuanto a calidad, rapidez, flexibilidad, funcionalidad y costos competitivos han puesto a las organizaciones, independientemente de su tamaño, en un proceso de cambio acelerado tanto en sus procesos organizacionales y en las personas involucradas en las mismas.

En este contexto, las tendencias en el campo de la información y avances en telecomunicaciones así como en la llamadas nuevas filosofías, métodos, técnicas que apuntan hacia un futuro en el que las habilidades, destrezas, competencias y actitudes de las personas deben responder de manera innovadora, rápida y eficiente antes los cambios que emergen, ya que la supervivencia de las mismas dependerá de los cambios en el entorno global.

Las personas se han convertido en estos últimos tiempos en el eje fundamental de la ventaja competitiva para las organizaciones, tomando en cuenta que el éxito de ellas depende no solo de sus líderes, sino en gran medida de la participación e integración de todos sus colaboradores.

Las empresas u organizaciones requieren una nueva forma de gestión para adaptarse a los cambios y sobrevivir. Una de estas es el empowerment, una nueva forma de orientar el trabajo de las organizaciones hacia un futuro próspero e innovador.

\section{UNA VISIÓN EMERGENTE}

Un ambiente competitivo, dinámico y complejo, donde la necesidad de innovación es un factor determinante para el éxito de las organizaciones, exige que las personas estén cada vez más involucradas en procesos de decisión asociados a las estrategias organizacionales (Ribeiro \& Almada, 2001).

Es ahí donde nace el empowerment, con el objetivo de capacitar a las personas para esos procesos decisorios en la organización, buscando 
como resultado las transformación de las empresas para adaptarlas a ambientes competitivos, complejos y dinámicos (Ribeiro \& Almada, 2001).

A lo largo de los años, diferentes abordajes han sido particularmente influyentes en diferentes momentos (Figura 1).

\section{Figura I}

Los diferentes enfoques del trabajo: cada una implica un equilibrio diferente entre control y compromiso (Slack, Chambers, Harland, Harrison \& Johnston, 1997, p.312).

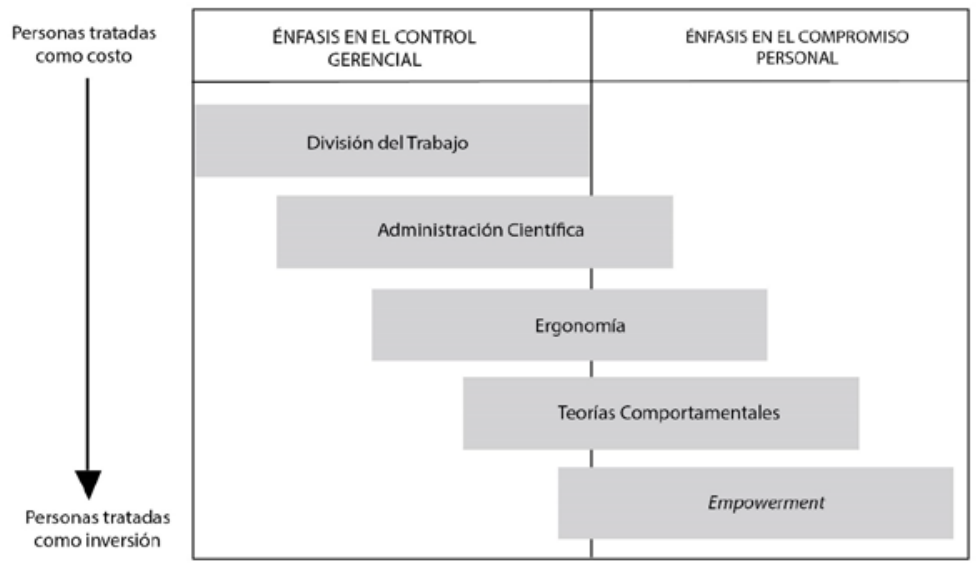

En la década de los 20, las ideas de Taylor, padre de la administración científica, influenciaron en la forma de pensar de los administradores sobre los mejores métodos para la realización del trabajo, donde había una separación entre la toma de decisión de los administradores y la ejecución hecha por los trabajadores de forma sumisa, sin embargo, a medida que generaba un aumento en términos productivos, generaba enajenación, conflicto y ausentismo (Wilkinson, 1998).

Emerge un nuevo paradigma, enfocado en la desburocratización, flexibilidad e innovación. En la década de los 90, la racionalización y el downsizing exigieron que las organizaciones sean más rápidas y ágiles que antes, haciendo que el relacionamiento burocrático entre los administradores y trabajadores se transforme; en este contexto, el empowerment es inevitable para asignar los sobrevivientes en la nueva organización (Wilkinson, 1998). 


\section{EMPOWERMENT}

\subsection{Concepto}

La dura competencia en la actualidad, la globalización de los mercados y las economías que fluctúan impredeciblemente, han llevado a las empresas en todo el mundo a buscar soluciones empresariales basados es nuevas y modernas teorías que mejoren su desempeño de manera global. Los presidentes de las organizaciones, según Salazar y Molano (2000), definen esta situación como un torneo de grandes ligas, de grandes competencias mundiales, el cual plantea cada día para las organizaciones, nuevos desafíos para los administradores.

En esta óptica, el empowerment surge como una solución a los viejos problemas del taylorismo y de la burocracia en los locales de trabajo, donde la creatividad era reprimida y los trabajadores se convertían en objetos de venta (Wilkinson, 1998).

Debido a esta situación, las empresas en la actualidad revisan constantemente su orientación organizacional para lograr sus objetivos establecidos, lo que implica que éstas tengan que desarrollar la capacidad de crear equipos de trabajo, estimular la comunicación, promover la lealtad, el compromiso, facilitar el consenso, entre otros, para lo cual se trasladan a otros ámbitos para adoptar técnicas y transferirlas a los sistemas y procesos organizacionales (Useche, 2004).

Salazar y Molano (2000) afirman que es indispensable tener sentido de trabajo en equipo, ya que se llega a ser un campeón cuando hay sincronía en todas las partes de la organización y se juega como una unidad, cuando el sentido del equipo está por encima de lo individual y todo el grupo trabaja por la convicción de su interdependencia, entendida esta como la fusión de los esfuerzos de cada uno para lograr una meta común.

Es así que, cada vez más, las empresas facultan al fomentar la formación de trabajo en equipo como equipos interfuncionales de calidad, grupos de enfoques concentrados en los clientes y equipos integrados de desarrollo de productos (Ronquillo, 2006).

En este sentido, las modernas organizaciones están dejando atrás las estructuras tradicionales organizadas en órganos y puestos y prefieren utilizar estructuras integradas por equipos, hoy en día las organizaciones están juntando e integrando a su personal en lugar de separarlos e individualizar el trabajo. Chiavenato (2009) insiste en que hoy en día los negocios están caracterizados por cambios, incertidumbre y una enorme competitividad, dejando en el olvido el diseño tradicional de las organizaciones por haber dejado de ofrecer adaptabilidad y flexibilidad. 
Así también, Dessler y Varela (2011) sugieren que el trabajo mismo en la actualidad se organiza cada vez más alrededor de equipos y procesos en vez de funciones especializadas.

La delegación de funciones a las personas de todas las áreas, así como la implantación de una cultura participativa es la base de las organizaciones que buscan descentralizar el poder e información entre sus miembros; el mismo Chiavenato (2009) enuncia el empowerment como el facultamiento en la toma de decisiones o delegación de autoridad.

Por su parte, Robinson (1998) afirma que el empowerment es un proceso que mejorará la efectividad y el desempeño en los negocios, introducirá cambios significativos en la cultura y clima de la organización y maximizará la utilización de las diferentes capacidades de la gente; es un proceso estratégico que busca una relación de socios entre la organización y su gente.

Así mismo, Murrell y Meredith (2002) expresan que el empowerment es la distribución creativa del poder y que es necesario diferenciar este término porque el poder es a veces entendido como fuerza o autoridad, lo cual implica que alguien tiene el control sobre un tercero, y en términos de empowerment empresarial, esto no tiene nada que ver sobre la fuerza de un individuo sobre otro, sino la suma de poder individual para que sume en beneficios comunes.

Resulta difícil encontrar una definición ideal del empowerment, pues es un concepto muy amplio que intenta establecer sus bases en la capacitación de los colaboradores mediante el otorgamiento de conocimientos propios de su labor, mejores condiciones de trabajo, un mejor flujo de información respecto a las estrategias de la organización y una modificación en la estructura piramidal de la empresa. En tanto que, su objetivo primordial es que los empleados se sientan más satisfechos con su trabajo y esto derive en un mejor rendimiento (Friedman, 1992).

Empowerment es un término en inglés que quiere decir potenciación o empoderamiento, el cual se traduce al hecho de delegar poder y autoridad a los subordinados y de conferirles el sentimiento de ser dueños de su propio trabajo (Koontz \& Weihrich, 1998).

Así, el empowerment significa dar poder a los empleados para aprovechar al máximo el talento colectivo, el secreto consiste en utilizar todo el personal, todas las habilidades, todo el tiempo, dar autoridad y recursos a las personas y dejarlas actuar (Chiavenato, 2009).

Según el mismo autor, hay tres principios esenciales para dar empowerment a las personas: 
- Dar a las personas un trabajo que consideren importante;

- Dar a las personas autoridad y responsabilidad plenas, independencia y autonomía en sus tareas y recursos;

- Permitir a las personas tomar decisiones respecto a su propio trabajo.

\section{Figura II}

Bases del empowerment (Chiavenato, 2009, p.195).

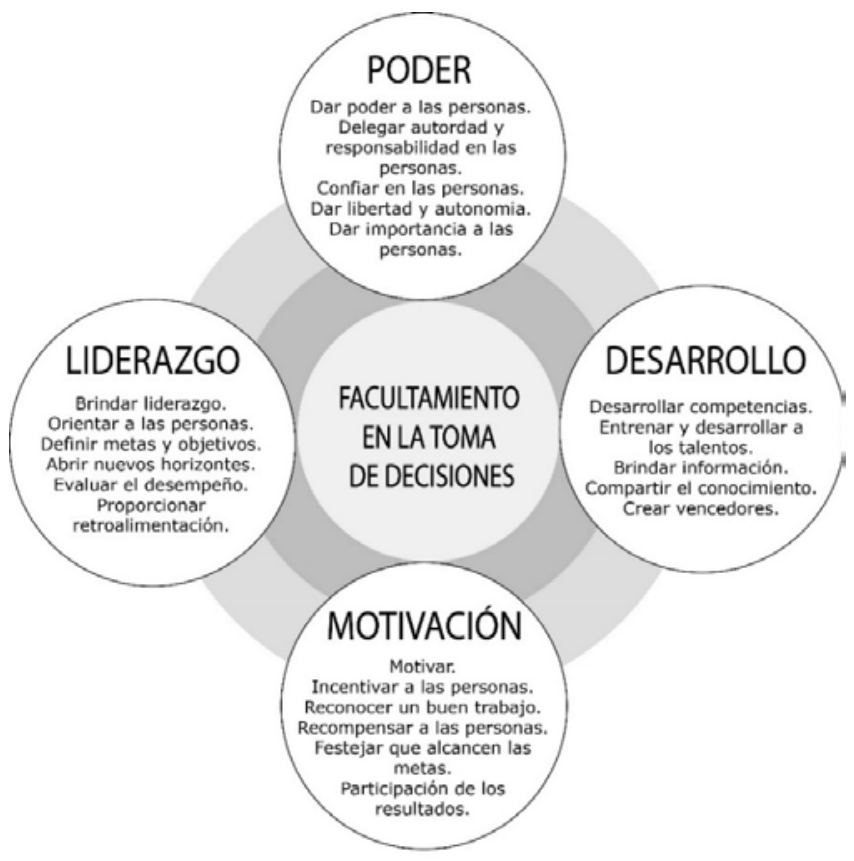

\subsection{Beneficios}

Para Schultz (2008), el empowerment mejora la inversión en capital humano y el rendimiento en el trabajo de tres formas:

- La primera es directa: resulta probable que los individuos autónomos, provistos de información y libres para hacer su trabajo, rindan mejor que otros trabajadores estrechamente controlados y menos informados.

- La segunda: es que la libertad y el control de trabajo que acompañan a la autonomía constituye un beneficio intrínseco de la inversión en capital humano que en la mayor parte de los casos, 
determina un incremento del esfuerzo. En consecuencia, tanto el trabajador como la compañía progresarán cuando aumente la autonomía (poder y responsabilidad).

- La tercera: sobreviene como un coste asociado con la gestión.

Toda organización que en determinado momento decida otorgar a sus colaboradores una mayor capacidad de decisión y autogestión, ha de realizarla mediante la adopción de una filosofía general que sea fácil de entender y que se comunique de manera sencilla. Esto es fundamental debido a la importancia que tiene el hecho de que los empleados deben ser conscientes de los cambios que se ejecutan, para que los valoren de manera positiva y puedan llevarlos a cabo en el menor tiempo posible y de la forma más eficiente (Wilson, 1996).

\subsection{Fundamentos y Aplicaciones}

De acuerdo con Spreitzer (2008), actualmente, más del 70\% de las organizaciones adoptan algún tipo de iniciativa de empowerment en por lo menos una parte de su fuerza de trabajo. Para alcanzar el éxito en el contexto competitivo actual, las organizaciones necesitan del conocimiento, de las ideas, de la creatividad y de la energía de cada colaborador.

En este sentido, se puede afirmar que, el papel de la alta gerencia es crear las condiciones necesarias para la eficacia del trabajo, asegurando que los colaboradores tengan acceso a información, apoyo y a recursos necesarios para la ejecución de sus tareas y, a las oportunidades para el desarrollo y crecimiento personal y profesional. Como consecuencia de elevados niveles de empowerment, los colaboradores experimentarán sentimientos positivos en su trabajo y serán más eficaces para alcanzar las metas organizacionales (Gouveia, 2013).

El empowerment permite que los colaboradores sean motivados por los gerentes para actuar, posibilitando así un mayor comprometimiento con la organización, niveles más elevados de confianza en la gestión, una mayor responsabilidad por el propio trabajo y menor propensión al estrés laboral y, como consecuencia, serán capaces de realizar el trabajo con mayor calidad, éxito y confianza. En otras palabas, cuando el trabajo está estructurado de tal forma que los colaboradores sientan el empowerment, estos responden en conformidad a la altura de los desafíos de la organización, observándose una mejoría en las actitudes de los trabajadores y un aumento de la eficacia y productividad organizacional (Laschinger, Finegan, Shamian, \& Wilk, 2001). A medida que los 
gerentes puedan crear un contexto de empowerment en las organizaciones, los colaboradores tienen el poder de decidir estar o no empoderados, debiendo sentirse ligados a la organización, confiantes de sus competencias y capacidades de influenciar el sistema en que están inmersos (Quinn \& Spreitzer, 1997).

El empowerment entonces es un proceso que se inicia con los gerentes y directivos de la organización, los cuales captan las ideas y deseos de los colaboradores, realizando una modificación en las políticas y estructuras para adaptarlas a las ideas y deseos de los empleados. Esto se hace con el fin de permitir que potencien sus talentos y habilidades para conseguir que los trabajadores se sientan cómodos en el desarrollo de sus habilidades aplicadas a sus respectivas labores, lo que provoca que la organización tenga mejores beneficios gracias al buen hacer de los trabajadores; todo esto afecta directamente a los gerentes y directivos de la organización, de manera que, construir una organización más efectiva repercute directamente sobre sus propias metas de trabajo y desarrollo profesional (Wilson, 1996).

En la siguiente figura, se expone las fases del empowerment según el mismo autor: 
Figura III

Círculo del empowerment (Wilson, 1996, p. 24).

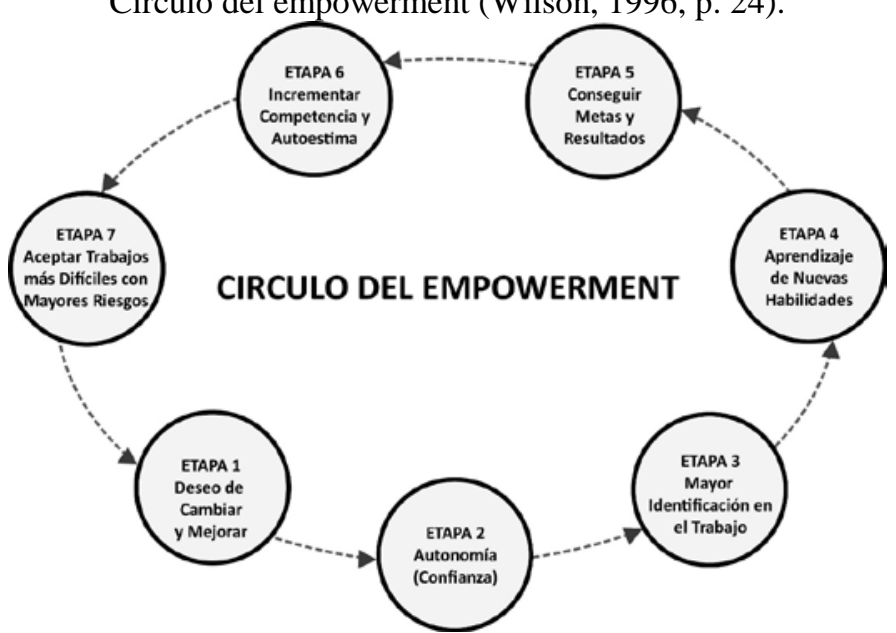

La atracción que supone el empowerment para los individuos y empresas, enmascara muchos de los factores que ayudan o impiden su introducción efectiva en las empresas. Se da por sentado que los beneficios del empowerment son tan importantes que las personas se convierten de inmediato en discípulos. Sin embargo, cualquier empresa que considera seriamente la idea de introducir esta herramienta debe pensar y evaluar algunos factores que ayudan a su efectiva introducción (Wilson, 1996).

\section{SÍNTESIS CONCLUSIVA}

En una organización que desarrolla el empowerment, sus empleados participan como socios, demostrando capacidad de trabajo tanto individual como colectivo, tomando la iniciativa y manejando autoridad a la hora de tomar decisiones estratégicas; con su utilización, las personas sentirán que, además de ser escuchadas sus ideas, podrán ser ejecutadas (McFarland, Senn, \& Childress, 1996). Según Alsop y Heinsohn (2005), si un individuo o grupo se empodera, ostenta la capacidad de tomar decisiones efectivamente.

Para alcanzar el empowerment es indispensable una estructura organizacional plana, donde se facilite el liderazgo, se manejen niveles de comunicación abiertos y honestos, relaciones de alianzas en pensamientos críticos, que sean flexibles tanto para el aprendizaje como para la toma de decisiones, retroalimentación sobre el desempeño y sistemas de 
incentivos, factores que conectados busquen transformar a los empleados en gestores de su propio puesto de trabajo (McLagan \& Nel, 1997).

Finalmente, el empowerment es un concepto que emerge en estos tiempos modernos como una poderosa herramienta para que las empresas puedan mantenerse en un mercado competitivo y globalizado; el empowerment busca obtener lo mejor de las personas, convertirlas en una fuerza colaborativa y transformar la organización en un sistema flexible, dinámico e innovador.

\section{FUENTES Y BIBLIOGRAFIA}

Alsop, R., \& Heinsohn, N. (2005). Measuring Empowerment: Structural Analysis and Framing Indicators. Washington, DC: Banco Mundial. Chiavenato, I. (2009). Gestión del Talento Humano (3a. Edición). México: McGraw Hill.

Dessler, G., \& Varela, R. (2011). Administración de Recursos Humanos: Enfoque Latinoamericano (5a. Edición). México: Pearson Prentice Hall.

Friedman, J. (1992). Empowerment: the politics of alterntive developement. Boston: Blackwell.

Gouveia, A. (2013). Efeitos do empowerment estrutural nos comportamentos de mobilização de profissionais de saúde e na percepção dos eventos adversos associados aos cuidados aos doentes: uma revisão sistemática da literatura (Tesse de Mestrado em Psicología das organizações e do trabalho). Universidade de Coimbra, Portugal.

Laschinger, H., Finegan, J., Shamian, J., \& Wilk, P. (2001). Impact of Structural and Psychological Empowerment on Job Strain in Nursing Work Settings: Expanding Kanter's Model. Journal of Advanced Nursing, 31(5), 260-272.

McFarland, L., Senn, L., \& Childress, J. (1996). Liderazgo para el siglo XXI. Colombia: McGraw Hill.

McLagan, P., \& Nel, C. (1997). The Age of Participation: New Governance for the Workplace and the World. San Francisco, CA: Berrett-Koehler.

Murrell, K., \& Meredith, M. (2002). Empowerment para su equipo. España: McGraw Hill.

Quinn, R. E., \& Spreitzer, G. M. (1997). The road to empowerment:

Seven questions every leader should consider. Organizational Dynamics, 26(2), 37-49. 
Ribeiro, C., \& Almada, F. (2001). Empowerment: ciclo de implementação, dimensões e tipologia. Gestão e Produção, 8(3), 237249.

Robinson, R. (1998). Como crear empowerment. Bogotá: McGraw Hill.

Ronquillo, J. (2006). Administración básica de la empresa familiar: un enfoque práctico. Mexico: Panorama.

Salazar, G., \& Molano, M. (2000). Coaching en acción. Colombia: McGraw Hill.

Schultz, M. (2008). La influencia de los empleados y sus culturas: Esencia de Marca. Madrid: Editorial Empresarial.

Slack, N., Chambers, S., Harland, C., Harrison, A., \& Johnston, R. (1997). Administração da Produção. São Paulo: Atlas.

Spreitzer, G. (2008). Taking Stock: A Review of More Than Twenty Years of Research on Empowerment at Work. In J. Barling, \& C. Cooper, The SAGE Handbook of Organizational Behavior (pp. 5472). Londres: SAGE Publications.

Useche, M. (2004). El coaching desde una perspectiva epistemológica. Revista Ciencias Sociales, 105(3), 125-132.

Wilkinson, A. (1998). Empowerment: theory and practice. Personnel Review, 27(1), 40-56.

Wilson, T. (1996). Manual del Empowerment: Cómo conseguir lo mejor de sus colaboradores. Barcelona: Gestión 2000. 